\title{
Resveratrol Exerts Dosage-Dependent Effects on the Self-Renewal and Neural Differentiation of hUC-MSCs
}

\author{
Xinxin Wang ${ }^{1,3}$, Shanshan $\mathrm{Ma}^{2,3}$, Nan Meng ${ }^{1}$, Ning $\mathrm{Yao}^{2}$, Kun Zhang', Qinghua Lí', Yanting Zhang', \\ Qu Xing, ${ }^{2}$ Kang Han ${ }^{2}$, Jishi Song', Bo Yang ${ }^{1, *}$, and Fangxia Guan ${ }^{1,2, *}$
}

Resveratrol (RES) plays a critical role in the fate of cells and longevity of animals via activation of the sirtuins1 (SIRT1) gene. In the present study, we intend to investigate whether RES could promote the self-renewal and neurallineage differentiation in human umbilical cord derived MSCs (hUC-MSCs) in vitro at concentrations ranging from 0.1 to $10 \mu \mathrm{M}$, and whether it exerts the effects by modulating the SIRT1 signaling. Herein, we demonstrated that RES at the concentrations of $0.1,1$ and $2.5 \mu M$ could promote cell viability and proliferation, mitigate senescence and induce expression of SIRT1 and Proliferating Cell Nuclear Antigen (PCNA) while inhibit the expression of p53 and p16. However, the effects were reversed by 5 and $10 \mu M$ of RES. Furthermore, RES could promote neural differentiation in a dose-dependent manner as evidenced by morphological changes and expression of neural markers (Nestin, $\beta$ III-tubulin and NSE), as well as pro-neural transcription factors Neurogenin (Ngn)1, Ngn2 and Mash1. Taken together, RES exerts a dosage-dependent effect on the self-renewal and neural differentiation of hUC-MSCs via SIRT1 signaling. The current study provides a new strategy to regulate the fate of hUC-MSCs and suggests a more favorable in vitro cell culture conditions for hUCMSCs-based therapies for some intractable neurological disorders.

\section{INTRODUCTION}

Mesenchymal stem cells (MSCs) have been spotlighted in the neural regenerative medicine (Can and Karahuseyinoglu, 2007).

${ }^{1}$ The First Affiliated Hospital of Zhengzhou University, Zhengzhou 450052, Henan Province, China, ${ }^{2}$ School of Life Sciences, Zhengzhou University, Zhengzhou 450001, Henan Province, China, ${ }^{3}$ These authors contributed equally to this work.

*Correspondence: guanfangxia@126.com (FG); yangbo96@126.com (BY)

Received 16 December, 2015; revised 24 March, 2016; accepted 6 April, 2016; published online 25 April, 2016

Keywords: human umbilical cord derived mesenchymal stem cells, neural differentiation, resveratrol, self-renewal, SIRT1
However, MSCs become senescent after consecutively passage in vitro as evidenced by morphological changes, low selfrenewal and neuronal differentiation rates (Lee et al., 2010). Therefore, it is critical to develop favorable culture conditions and direct the fate of stem cells for specialized therapy.

Sirt1 gene is a member of the Sirtuins family which possess histone deacetylase activity and plays an important role in regulating the aging of cells and neurodegenerative diseases. However, the protein level of SIRT1 declines with MSCs passage in vitro (Chen et al., 2014). Resveratrol (RES, 3, 5, 4, 9hydroxystilbene), a natural polyphenol compound abundant in grapes, peanuts, blueberries and many other plants (Chen et al., 2015; Guo et al., 2014), is an effective activator of Sirt1 (Ozcan et al., 2015). Numerous in vitro and in vivo studies have shown that RES plays a pivotal role in cell proliferation, apoptosis, senescence and differentiation, anticancer, neuroprotection, immunomodulation by regulating SIRT1 signaling (Atkins et al., 2014; Simic et al., 2013; Zhang et al., 2015). However, the role of RES/SIRT1 on different cells remains controversial. Some studies demonstrated that RES could enhance cell survival, proliferation and multi-potent progenitor capacity, prevent senescence in cultured primary human keratinocytes, bone marrow cells and other cell types (Ido et al., 2015; Rimmele et al., 2014). Whereas, some other evidences showed that RES could trigger cell cycle arrest, apoptosis, senescence and inhibit proliferation of cancer cells (Jackson et al., 2016; Zhu et al., 2015) and vascular smooth muscle cells (Guo et al., 2014).

Since the controversial effects of RES on different cells retard the research progress and limit its clinical application, this study aims to investigate the role of RES in the fate of human umbilical cord derived MSCs (hUC-MSCs) in vitro. We will examine the viability, proliferation, cell cycle, apoptosis, senescence, neural differentiation, as well as the SIRT1 signaling of hUCMSCs exposed to different concentrations of RES. This study will enlighten the anti-aging effect of RES and promote the application of RES modified hUC-MSCs in neural regeneration, contributing to efficient stem-cell based therapy for neural injury and neural degenerative disorders.

\section{MATERIALS AND METHODS}

Isolation and culture of hUC-MSCs

hUC-MSCs were isolated as previously described (Ma et al., 
2014). The cultured cells were harvested at passage 4 (P4) for a typical experiment. The study was approved by the Ethics Committees of the First Affiliated Hospital of Zhengzhou University and consented by the donors of umbilical cord.

\section{Identification of hUC-MSCs by flowcytometry}

hUC-MSCs were harvested and washed with ice-cold PBS. Cells were labeled with the following antibodies: CD29-PE, CD44-FITC, HLA-ABC-FITC, HLA-DR-FITC, CD34-PE, CD45PE, CD51-FITC, and CD105-PE (BD Bioscience, USA) before analyzed by the FACS Calibur flow cytometer (Becton-Dickinson, USA).

\section{Administration of RES}

RES $50 \mu \mathrm{M}$ stock solution (trans-3,4,5-trihydroxystilbene; R5010; Sigma, USA) was suspended in DMSO (Sigma, USA) and diluted to specific concentration before use. The final working concentration of DMSO was less than $0.1 \%$ for the in vitro experiment. hUC-MSCs were subjected to different doses of RES in the following experiments.

\section{CCK-8 assay}

Cell viability was quantitatively determined by a CCK-8 kit (Dojindo Molecular Technologies, Japan) according to the manufacture's protocol. Briefly, hUC-MSCs at P4 were plated in 96-well plate at a density of 1000 cells/well and cultured in $100 \mu \mathrm{M}$ DMEM/F12 with RES $(0.1,1,2.5,5,10,20,50$ and $100 \mu \mathrm{M})$ for $1,3,6,8,10$ and 12 days, rinsed with PBS and incubated in DMEM/F12 with $10 \mu \mathrm{l} \mathrm{CCK-8} \mathrm{for} \mathrm{each} \mathrm{well} \mathrm{for} 2$ $\mathrm{h}$ at $37^{\circ} \mathrm{C}$ and the absorbance (OD) of the solution was measured by a microplate reader (Bio-Rad, USA) at a wavelength of $450 \mathrm{~nm}$.

\section{EdU labeling}

To examine the effect of RES on the proliferation rate of hUCMSCs, cells at P4 were seeded in 24-well plate (8000 cells/ well) for 24 hours to allow for stabilization and then exposed to RES $(0,0.1,1,2.5,5$ and $10 \mu \mathrm{M})$ for 6 days before EdU (Ribobio, China) was added. The EdU labeling duration was determined at $24 \mathrm{~h}$ according to the average cell-doubling time for hUC-MSCs. Images were visualized using fluorescent microscope (Olympus, Japan). The red (EdU-labeled) and blue (Hoechst-labeled) cells were counted.

\section{Senesence associated $\beta$-galactosidase staining}

hUC-MSCs at P4 were plated at similar density in their respective media and cultured for 6 days before senescence-associated $\beta$ galactosidase (SA- $\beta$-gal) staining (Beyotime, China). Briefly, cells were fixed in $4 \%(\mathrm{v} / \mathrm{v})$ formaldehyde for $10 \mathrm{~min}$ before stained with SA- $\beta$-gal-staining solution at $\mathrm{pH} 6.0$ for $12 \mathrm{~h}$. The SA- $\beta$-gal-positive cells exhibited blue color. The number of positive cells was counted under a phase-contrast microscope. The experiments were carried out in triplicate.

\section{Cell cycle detection}

hUC-MSCs at P4 were incubated with RES (0, 0.1, 1, 2.5, 5 and $10 \mu \mathrm{M}$ ) for 6 days before propidium iodide (PI) staining performed as previously described (Ma et al., 2014).

\section{Cell apoptosis detection}

Cell apoptosis was analyzed by the FITC Annexin V Apoptosis Detection Kit I (BD Biosciences, USA) following the manufacturer's instructions. Briefly, hUC-MSCs at P4 were incubated with RES $(0,0.1,1,2.5,5$ and $10 \mu \mathrm{M})$ for 6 days and then col- lected for FITC- Annexin V and PI staining as previously reported (Zhu et al., 2016).

\section{Neural differentiation}

hUC-MSCs at P4 were incubated with RES $(0,0.1,1,2.5,5$ and $10 \mu \mathrm{M})$ for 6 days before neural differentiation induction, which was modified on the basis of the protocol previously described (Karahuseyinoglu et al., 2007). hUC-MSCs were pre-induced for $24 \mathrm{~h}$ in DMEM-LG containing $20 \%$ fetal bovine serum (FBS), $10 \mathrm{ng} / \mathrm{ml}$ basic fibroblast growth factor (bFGF, Peprotech, USA) was added for an additional $24 \mathrm{~h}$, then incubated in the induction medium for another $24 \mathrm{~h}$ : DMEM-LG with $2 \%$ DMSO (Sigma, USA), $100 \mu$ M butylated hydroxyanisole (Sigma, USA), $25 \mathrm{mM} \mathrm{KCl}, 10 \mu \mathrm{M}$ forskolin, $5 \mu \mathrm{g} / \mathrm{ml}$ insulin and $1 \mu \mathrm{M}$ hydrocortisone, followed by neurobasal medium, supplemented with $10 \% \mathrm{FBS}, 10 \mathrm{ng} / \mathrm{ml}$ epidermal growth factor (Sigma, USA), $10 \mathrm{ng} / \mathrm{ml} b F G F, 1 \times \mathrm{N}_{2}$ supplement (Gibco, USA), $1 \times$ B-27 supplement (Gibco, USA), and $2 \mathrm{mM}$ L-glutamine (Sigma, USA) for the maintenance of differentiation.

\section{Immunofluorence staining}

The $1 \times 10^{5}$ cells were plated on 24 -well plate and after rinsing with PBS, cells were fixed with $4 \%$ paraformaldehyde for 10 min at room temperature. After blocking with $10 \%$ normal goat serum, cells were incubated overnight at $4^{\circ} \mathrm{C}$ with specific antibodies against Nestin (1:50, SantaCruz, USA), $\beta$ III-tubulin (1:100, Cell Signaling Technology, USA) or Neuron Specific Enolase (NSE, 1:3,000, Abcam, USA). After rinsing in PBS three times, cells were incubated for 1 hour at room temperature in Cy3-conjugated anti-mouse/-rabbit anti IgG $(1: 1,000$, Molecular Probes, USA) for visualization, followed by DAPI staining (Biotech, China). Immunofluorescent images were visualized with fluorescence microscopy. The negative control was incubated with PBS instead of primary antibody, and no immunoreactivity was observed.

\section{Quantitative real-time polymerase chain reaction (qRT-PCR)}

Total RNA was isolated using Trizol reagent (Invitrogen) according to the instructions, and mRNA was reverse transcribed using PrimeScript ${ }^{T M}$ RT-PCR Kit (TaKaRa, Japan). qRT-PCR were performed on an ABI 7500 real-time PCR system (Thermo Fisher Scientific, USA) using a SYBR Premix Ex Taq ${ }^{\mathrm{TM}}$ (Prefect Real Time, Takara, Japan). Glyceral dehyde 3-phosphate dehydrogenase (GAPDH) was employed as the internal standard. Relative expression levels of different genes were calculated using the $2^{-\Delta \Lambda C t}$. The sequences of primers for qRT-PCR were shown in Table 1.

\section{Western blotting}

Cells were incubated with RES of the indicated concentrations above before Western Blotting, which was performed as previously described (Ma et al., 2014). Primary antibodies (SIRT1, 1:1000; PCNA, 1:1000; p53,1:1000; p21,1:500; p16,1:500, cell signaling technology, USA) were employed.

\section{Statistical analysis}

Data were expressed as mean \pm standard deviation (SD). Analysis of Variance (ANOVA) followed by LSD test were employed to determine the significance between different groups. $p<0.05$ was considered statistically significant. Data were representative of three independent experiments. All data analyses were using SPSS 18.0 statistical software. 
Table 1. Sequences of primers for qRT-PCR

\begin{tabular}{lll}
\hline Gene & Sequence & bp \\
\hline Ngn1 & 5'-CCAAAGACTTGCTCCACACA-3' (F) & 164 \\
& 5'-CTTTAAAGCTCCCGCTTCCT-3' (R) & \\
Ngn2 & 5'-CCTGGAAACCATCTCACTTCA-3' (F) & 81 \\
& 5'-TACCCAAAGCCAAGAAATGC-3' (R) & \\
Mash1 & 5'-CCAGTTGTACTTCAGCACC-3' (F) & 73 \\
& 5'-TGCCACTTTGAGTTTGGAC-3' (R) & \\
SIRT1 & 5'-GAGATAACCTTCTGTTCGGTGATGAA-3' (F) & 194 \\
& 5'-CGGCAATAAATCTTTAAGAATTGTTCG-3' (R) \\
PCNA & 5'-GTAGTAAAGATGCCTTCTGGTG-3' (F) & 190 \\
& 5'-TCTCTATGGTAACAGCTTCCTC-3' (R) & \\
p53 & 5'-CCGCAGTCAGATCCTAGCG-3' (F) & 118 \\
& 5'-AATCATCCATTGCTTGGGACG-3' (R) & \\
p21 & 5'-CCTGTCACTGTCTTGTACCCT-3' (F) & 130 \\
& 5'-GCGTTTGGAGTGGTAGAAATCT-3' (R) & \\
p16 & 5'-CTTGCCTGGAAAGATACCG-3' (F) & 94 \\
& 5'-CCCTCCTCTTTCTTCCTCC-3' (R) & \\
GAPDH & 5'-ACCCACTCCTCCACCTTTGA-3' (F) \\
& 5'-CTGTTGCTGTAGCCAAATTCGT-3' (R) & \\
\hline
\end{tabular}

\section{RESULTS}

\section{Morphologic and phenotypic characteristics of cultured} hUC-MSCs

hUC-MSCs grew out from the Wharton's jelly 7-10 days after isolation, and displayed a monolayer of bipolar spindle-like or fibroblast-like morphology with a whirlpool-like array. However, the cells underwent morphological changes and became flat, swollen and irregular as the passage number increased (Figs. 1A-1F). Flow cytometry revealed that hUC-MSCs were positive for stromal matrix markers (CD44, CD105), integrin markers (CD29, CD51), and HLA-ABC, while negative for hematopoietic lineage markers (CD34, CD45) and HLA-DR (data not shown).

\section{Effects of RES on the viability of hUC-MSCS}

We found that RES exhibited a dose-dependent promoting effect on the viability of hUC-MSCs at a concentration of $0.1,1$ and $2.5 \mu \mathrm{M}$ after incubating for 6 days $(p<0.05$, Fig. 2$)$. However, cell viability was inhibited after 6 days incubation of 5 and $10 \mu \mathrm{M}$ RES $(p<0.05)$, and in the presence of 20,50 and 100 $\mu \mathrm{M}$ RES, cell viability was significantly reduced beginning at the first day of RES stimulation $(p<0.05)$. Our results showed that RES exerted a dose-and time-dependent effect on the viability of hUC-MSCs (Fig. 2). The hUC-MSCs were pretreated with $0.1,1,2.5,5$ and $10 \mu \mathrm{M}$ RES for 6 days in the subsequent experiments.

\section{Effects of RES on the proliferation rate of hUC-MSCS}

The effect of RES on cell proliferation was analyzed by EdU staining at different concentrations and time points. As shown in Figs. 3A and 3B, 0.1, 1 and $2.5 \mu \mathrm{M}$ RES significantly increased the percentages of EdU-labeling cells, compared with the control. However, the proliferation of hUC-MSCs was significantly inhibited when cells were challenged with 5 and $10 \mu \mathrm{M}$ RES $(p$ $<0.05$ ), which confirmed the CCK-8 results of cell viability. Therefore, the results demonstrated that there was a dosage enhancing effect of $(0.1,1$ and $2.5 \mu \mathrm{M})$ RES on the proliferation rate of hUC-MSCs, culminating at $2.5 \mu \mathrm{M}$.
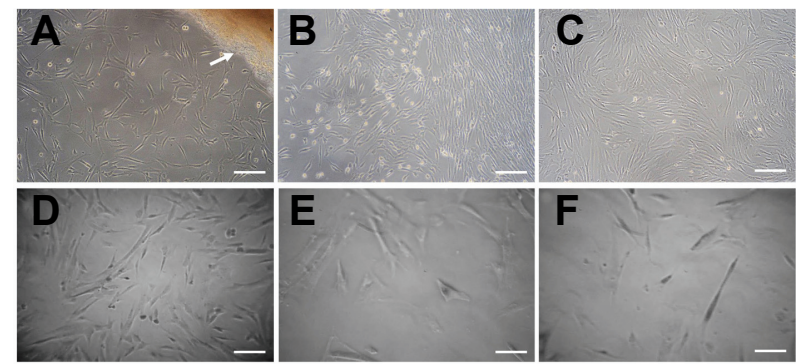

Fig. 1. The morphologic characteristics of hUC-MSC. (A) primary cells (P0); (B) P1; (C) P3 (D) P9; (E) P11; (F) P15. Arrow: Wharton jelly. Scale bars $=50 \mu \mathrm{m}$.

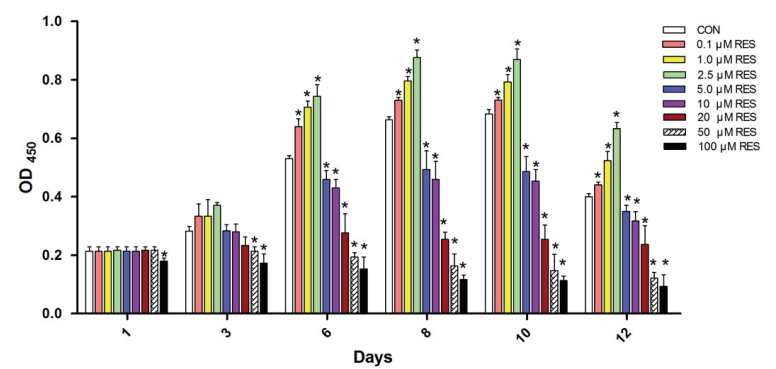

Fig. 2. RSE exerts dosage- and duration-dependent effect on the viability of hUC-MSCs. Cells were plated at equal density before challenged with different concentrations $(0.1,1,2.5,5,10,20,50$ and $100 \mu \mathrm{M}$ ) of RES, the $\mathrm{OD}_{450}$ values of the CCK-8 test assay were detected after RES incubation for $1,3,6,8,10$ and 12 days respectively. The error bars represent standard deviation (SD, triplicates in each treatment condition). Compared with the CON, the $\mathrm{OD}_{450}$ is significantly increased in the $0.1,1$ and $2.5 \mu \mathrm{M}$ RES groups 6 days later, in contrast, the $\mathrm{OD}_{450}$ significantly decreased 6 days later in the 5, $10 \mu \mathrm{M}$ RES groups, and 1 day later in the 20,50 and $100 \mu \mathrm{M}$ RES groups. CON: cells without RES incubation. ${ }^{*} p<0.05$ vs. CON.

\section{Effects of RES on the senescence of hUC-MSCs}

As shown in Figs. $3 \mathrm{C}$ and 3D, there were significantly less SA$\beta$-gal positive cells in the $0.1,1$ and $2.5 \mu \mathrm{M}$ RES treated groups $(p<0.05)$, whereas, significantly more cells stained positive for SA- $\beta$-gal activity in the 5 or $10 \mu \mathrm{M}$ RES groups $(p<0.05)$. These results indicated that $0.1,1$ and $2.5 \mu \mathrm{M}$ RES inhibited senescence, whereas 5 and $10 \mu \mathrm{M}$ RES accelerated senescence, with the most effective anti-senescence detected at $2.5 \mu \mathrm{M}$ RES.

Effects of RES on the cell cycle of hUC-MSCs

Flow cytometry was used to detect the increased percentages of cells in $S$ and $G_{2} / M$ phases (Fig. 3E). There was a reduction in the percentage in $\mathrm{G}_{0} / \mathrm{G}_{1}$ phase in hUC-MSCs treated with 0.1 , 1 and $2.5 \mu \mathrm{M}$ RES. However, there was no significant difference when compared with the control $(p<0.05)$. At a concentration of 5 and $10 \mu \mathrm{M}$ RES there was a significant arrest in the $S$ phase, with concomitant decrease in the percentage of cells in $\mathrm{G}_{0} / \mathrm{G}_{1}$ phase $(p<0.05$, Fig. 3E).

Effects of RES on the apoptosis of hUC-MSCs

Flow cytometric detection of Annexin-V-FITC in / Propidium 
A

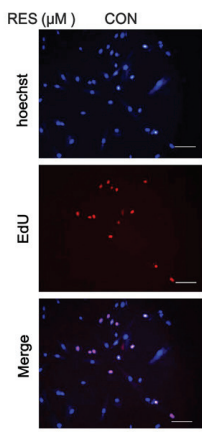

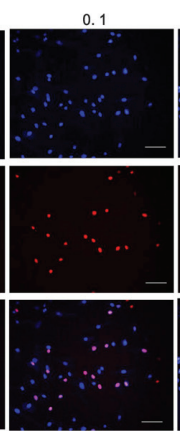
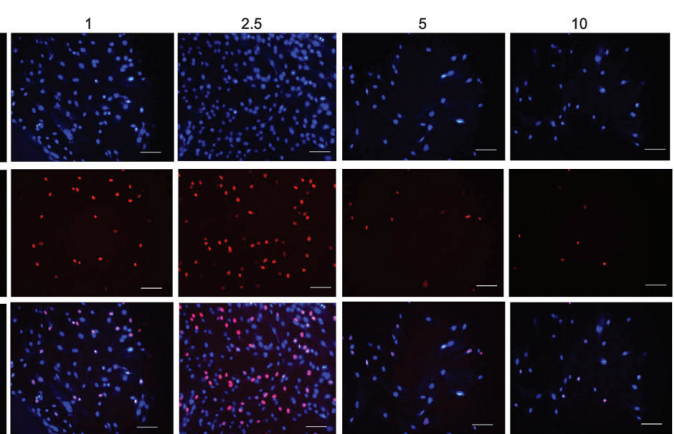

B

C
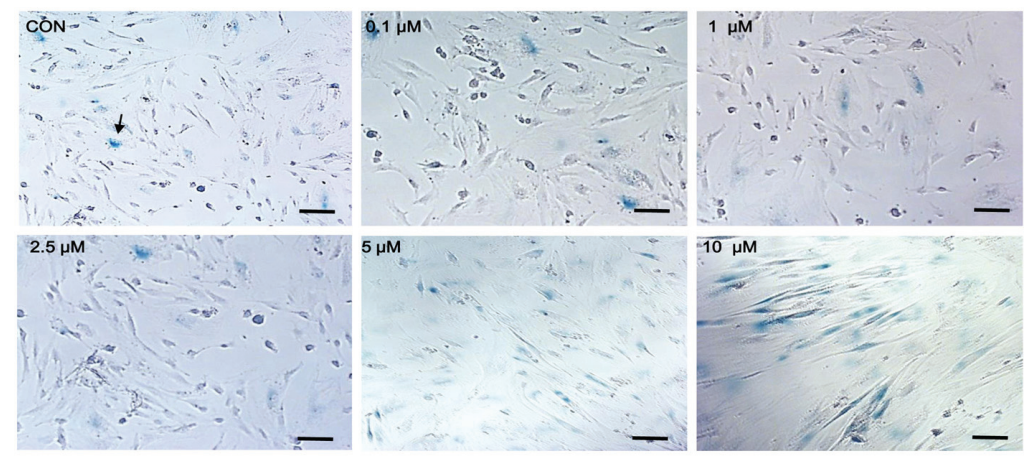

D

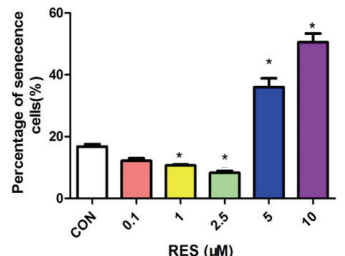

E
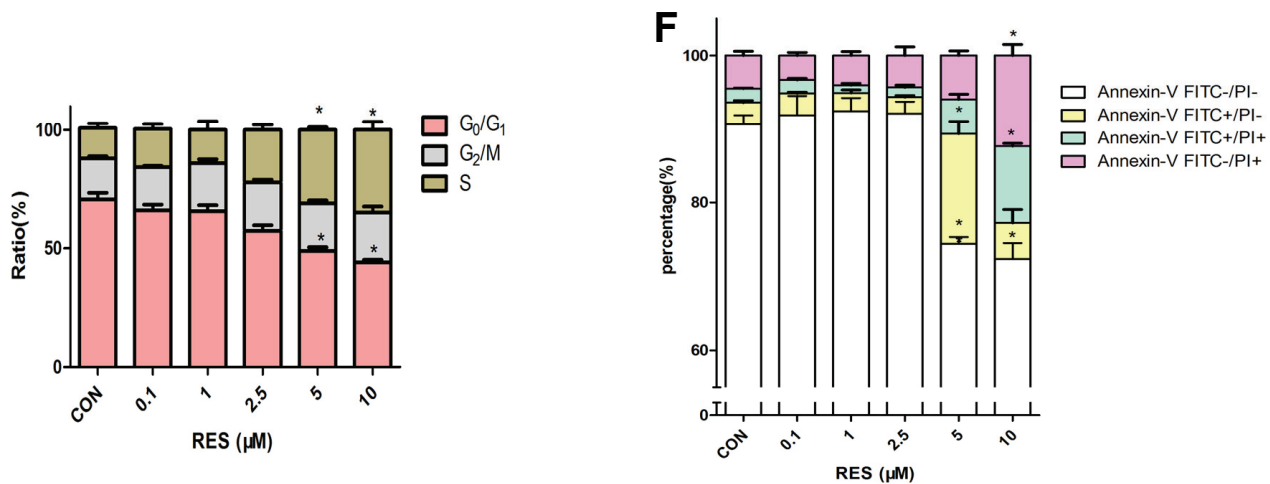

Fig. 3. RSE exerts concentration-dependent effect on the proliferation, senescence, cell cycle and apoptosis rate of hUC-MSCs. hUC-MSCs were pre-incubated with RES $(0.1,1,2.5,5$ and $10 \mu \mathrm{M})$ for 6 days before the detections. (A) Cells were exposed to EdU for $24 \mathrm{~h}$ and then fixed and stained. Images were taken using UV/Blue dual filter, and cells labeled with EdU were Red. Scale bar $=100 \mu \mathrm{m}$. (B) The percentage of EdU-labeled (proliferating) cells vs. total cells. (C) The SA- $\beta$-gal-positive cells exhibited blue color (indicated by arrows) under phase-contact microscope. Scale bar $=50 \mu \mathrm{m}$. (D) Column represents percentage of senescent cells vs. total cells based on the photos taken in each group. (E) RES increases the percentage of S-phase cells in a dosage-dependent manner. Stacked columns represent the relative distribution of cells in $S, G_{2} / M$ or $\left(S+G_{2} / M\right)$ phase detected by Flow cytometry. (F) Annexin V FITC / PI staining of the hUC-MSCs showed no significant change of apoptotic and necrotic cells in the $0.1,1$ and $2.5 \mu \mathrm{M}$ RES treated group $(p>0.05)$, while 5 and $10 \mu \mathrm{M}$ RES treatment resulted in significantly less AnnexinV FITC- / PI- cells, with concomitant higher percentages of AnnexinV FITC+/PI-, AnnexinV FITC+/PI+, and AnnexinV FITC-/PI+ cells $(p<0.05)$. Results were from three independent experiments. Error bars represent SD. CON: cells without RES incubation. ${ }^{*} p$ $<0.05$ vs. CON.

iodide (PI)staining revealed a dose-dependent downward trend in the percentages of apoptotic and necrotic cells among the RES $(0.1,1$ and $2.5 \mu \mathrm{M})$ treatment groups (Fig. 3F), although there was no significant difference when compared with the control $(p>0.05) .5$ and $10 \mu \mathrm{M}$ RES resulted in significantly lower percentage of Annexin V FITC-/PI- normal cells, with a concomitant higher percentages of Annexin V FITC+/PI-, An- nexin V FITC+/PI+ and Annexin V FITC-/PI+ cells $(p<0.05)$, indicating more apoptosis, necrotic and dead cells than the control group (Fig. 3F) .

Effects of RES on the neural differentiation of hUC-MSCS hUC-MSCs underwent neuronal differentiation by a multi-step protocol. Most differentiated hUC-MSCs demonstrated neural 
A
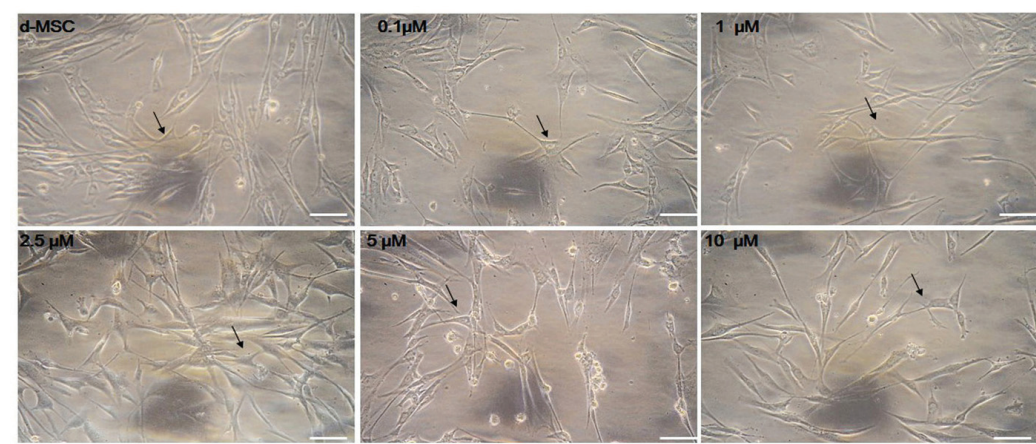

B

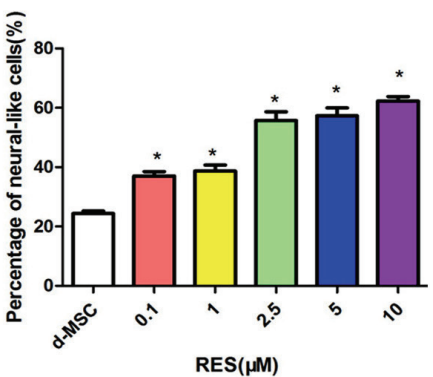

C
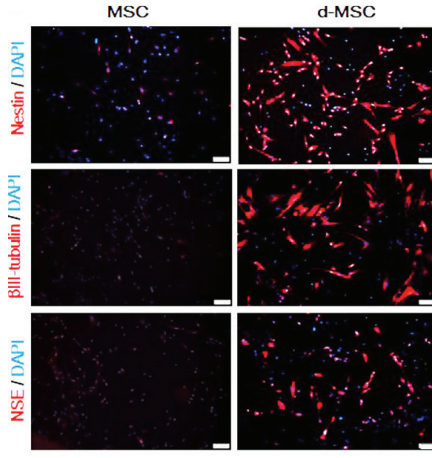

D

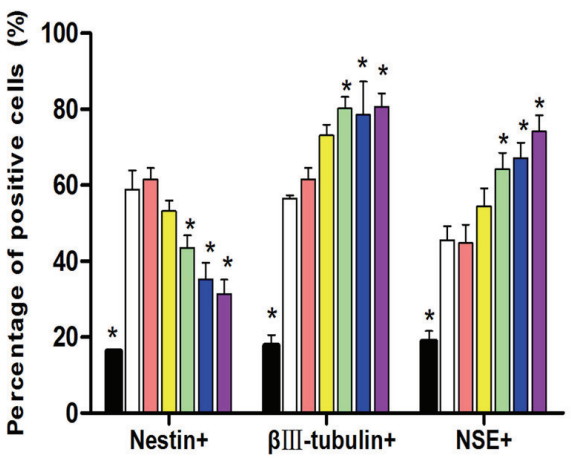

$0.1 \mu \mathrm{M}$
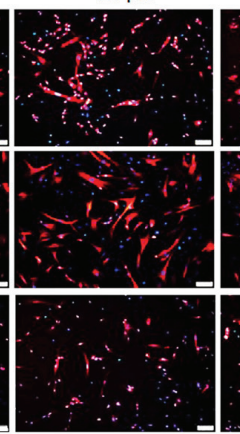

E

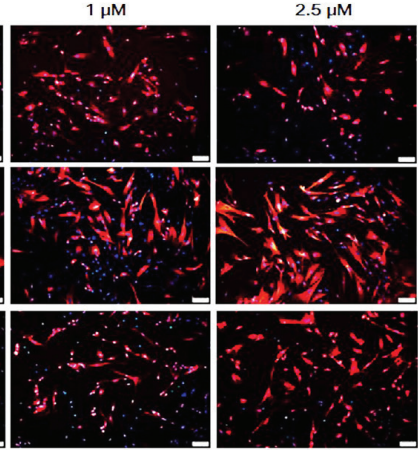

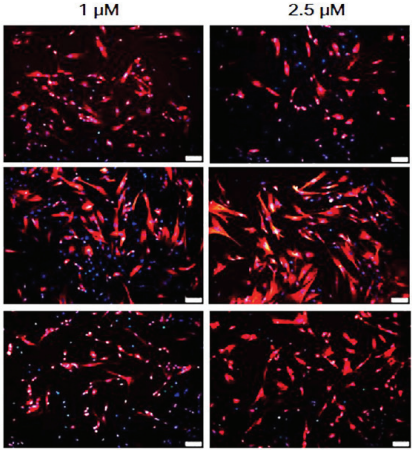
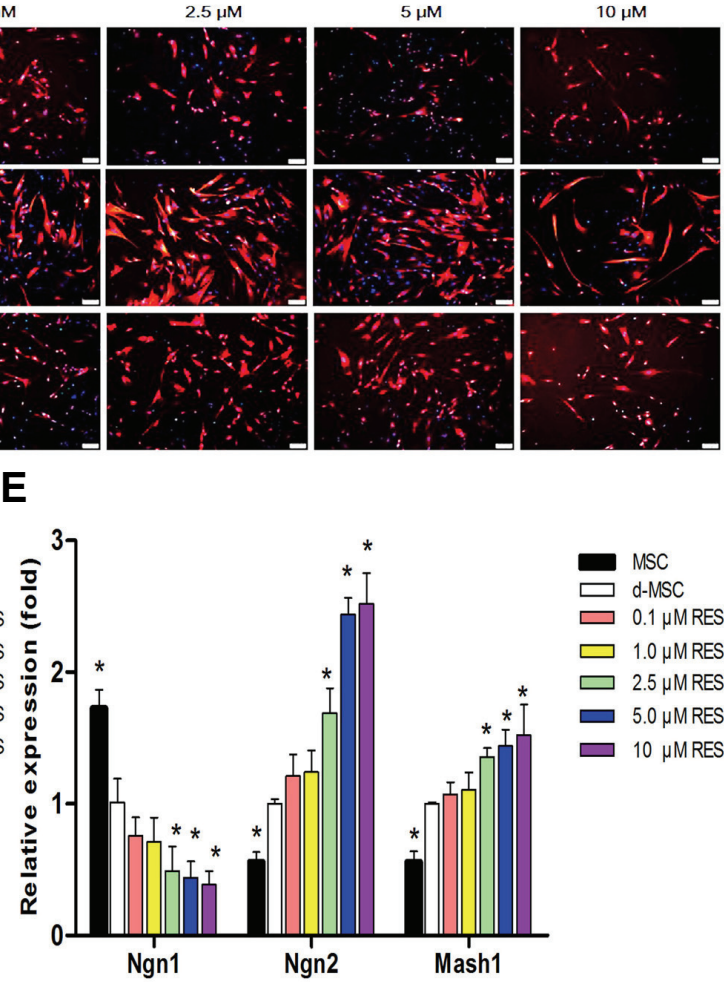

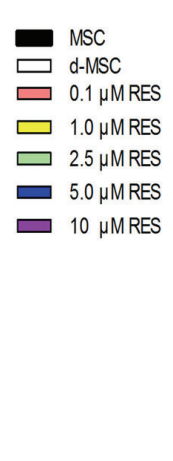

Fig. 4. RES stimulates neuronal lineage differentiation of hUC-MSCs. (A) Representative images of neural differentiation of hUC-MSCs preincubated with /without different doses of RES. Arrow: neuronal-shaped cells. Scale bar $=100 \mu \mathrm{m}$. (B) Quantitation of the neuronal differentiated rate by counting the neuronal-shaped cells and total cells. The results were presented as the mean \pm SD derived from three samples. (C) Representative immunofluorescence pictures of the expression of Nestin, $\beta$ III-tubulin and NSE (Red) in the hUC-MSCs with different RES treatment. Nuclei were counterstained with DAPI (blue). Scale bar $=100 \mu \mathrm{m}$. (D) Percentages of cells positive for specific neural markers (Nestin/ßIII-tubulin/NSE) vs. Total cells detected by immunofluorescence. (E) The relative gene expression of Ngn1, Ngn2 and Mash1 in the different groups, which normalized by the housekeeping gene GAPDH. Data are presented as the mean \pm SD derived from three independent experiments. Abbreviations: MSC: hUC-MSCs without neural induction. d-MSCs: hUC-MSCs underwent general neural differentiation induction without RES pre-incubation. ${ }^{*} p<0.05$ vs. CON.

appearance with retracted cell body, refractive karyon, protruded dendrites and axons after neural induction (Fig. 4A), while hUC-MSCs without induction retained long fusiform shape. RES pre-incubation facilitated the neural differentiation of hUCMSCs in all concentrations (Fig. 4A). There were more neuronal-shaped cells in RES-pretreated hUC-MSCs than that without pretreatment, especially at the concentration of $2.5,5$ and $10 \mu \mathrm{M}(p<0.05$, Fig. 4B). Immunoflurorescence of hUCMSCs without neural induction detected low expression of Nes- tin, $\beta$ III-tubulin and NSE. Pre-treated with $0.1,1,2.5,5$ and 10 $\mu \mathrm{M}$ RES before neural induction reduced the expression of Nestin, while up-regulated the expression of $\beta$ III-tubulin and NSE in a dose-depended manner (Figs. 4C and 4D).

RES regulates the expression of proneural transcription factors in hUC-MSCs

The qRT-PCR analysis revealed that after neural differentiation, the expression of Neurogenin1 (Ngn1) was decreased, while 
A

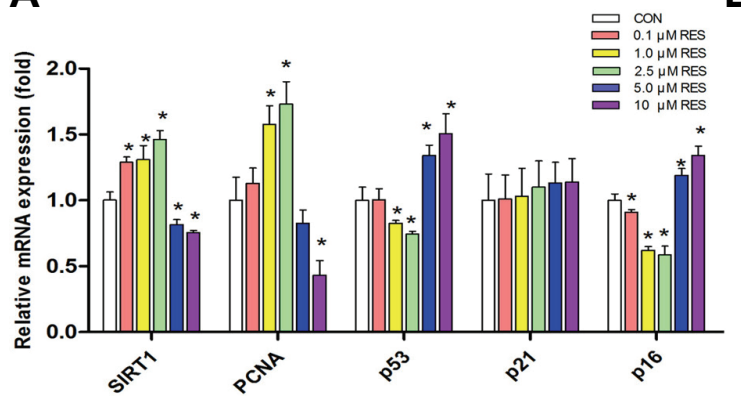

B

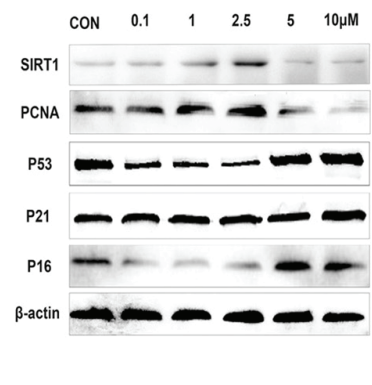

Fig. 5. RES regulates the gene and protein expression of SIRT1, PCNA, p53, p21, and p16. (A) qRT-PCR results of the mRNA level of SIRT1, PCNA, p53, p21 and p16 in RES treated cells was normalized by housekeeping gene GAPDH correspondingly and quantified relative to that of the control. Data shown are the mean values of three repeats. Error bars represent standard deviation. ${ }^{*} p<$ 0.05 vs. CON. (B) Western blot results of the expression of SIRT1, PCNA, p53, p21, p16 and internal control $\beta$-actin.
Ngn2 and Mash1 were increased $(p<0.05)$, and the effect was enhanced by RES in a dose-enhancing manner (Fig. 4E). These data indicate that $2.5,5$ and $10 \mu \mathrm{M}$ RES significantly promotes the neuronal differentiation of hUC-MSCs by regulating Ngn1, Ngn2 and Mash1 $(p<0.05)$.

\section{RES regulates SIRT1 signaling of hUC-MSCs}

To explore the molecular mechanism of RES on the cell viability, proliferation, cell cycle, apoptosis and senescence of hUCMSCs, we examined the expression of critical genes involved in those biological processes by qRT-PCR and Western Blot, including SIRT1, PCNA, p53, p21, and p16. As indicated in Fig. $5 \mathrm{~A}$, the expression of SIRT1 and PCNA in hUC-MSCs were significantly increased by $0.1,1$ or $2.5 \mu \mathrm{M}$ RES $(p<0.05)$, while inhibited by 5 or $10 \mu \mathrm{M}$ RES $(p<0.05)$. On the contrary, the expression of $\mathrm{p} 53$ and $\mathrm{p} 16$ were inhibited by $0.1,1$ or $2.5 \mu \mathrm{M}$ RES $(p<0.05)$ and enhanced by 5 or $10 \mu \mathrm{M}$ RES $(p<0.05)$. However, p21 underwent no significant change with RES treatment in the present study $(p>0.05)$. The protein expression detected by Western blot (Fig. 5B) was consistent with those of the qRT-PCR. Since SIRT1 is a critical player in antiaging, p53, p21 and p16 are involved in apoptosis and cell cycle inhibition (Luo et al., 2001; Zhang et al., 2011) and PCNA is a S-phase cell marker (Mikula-Pietrasik et al., 2012), our results indicated that $0.1,1$ and $2.5 \mu \mathrm{M}$ RES promotesed cell survival, proliferation and suppresses aging by stimulating SIRT and PCNA, while inhibiting p53 and p16 in vitro. Whereas, the S-phase cycle arrest and senescence induced by 5 and $10 \mu \mathrm{M}$ RES were associated with a decrease of SIRT1 and PCNA while increase of p53 and p16.

\section{DISCUSSION}

Regulating the self-renewal and lineage commitment of stem cells is the key factor in regenerative medicine. Studies have shown that RES promotes the survival of adipose-derived mesenchymal stem cells (Pinarli et al., 2013), enhance the proliferation and osteoblastic differentiation of human bone marrow-derived mesenchymal stem cells (Dai et al., 2007) and direct the neuronal differentiation of human bone marrow mesenchymal stem cells (Joe et al., 2015). However, some researchers argued that RES inhibited the proliferation of neural progenitor cells (Park et al., 2012), negatively regulated the neurogenic potential of neural precursors (Saharan et al., 2013). These results suggested that RES might play different or even opposite role in different cell type under specific milieu. Since hUC-MSCs are promising candidate for some intractable diseases, it is essential to gain better understanding of the effect of RES on hUC-MSCs development.
The present study revealed that incubation with $0.1,1$ and $2.5 \mu \mathrm{M}$ RES for 6 days in vitro facilitated the self-renewal of hUC-MSCs by enhancing cell viability and proliferation, mitigating senescence. Whereas, 5 and $10 \mu \mathrm{M}$ RES inhibited selfrenewal of hUC-MSCs by reducing cell viability and proliferation, increasing senescence and apoptosis and leading to cell cycle arrest in $\mathrm{S}$ and/or $\mathrm{G}_{2} / \mathrm{M}$ phase. In parallel with previous researches (Mikula-Pietrasik et al., 2012; Yuan et al., 2012), we suggest that RES exerts opposite effect on the self-renewal of hUC-MSCs through different regulation of cell viability, proliferation, senescence, cell cycle and apoptosis in a dose-dependent manner. This study shed light on the hypothesis that the destiny of stem cells could be directed by RES and provide theoretical and technical basis for further development of regenerative medicine.

In addition, we found that hUC-MSCs expressed low level of Nestin, BIII-tubulin and NSE, indicators of multi-potent neural stem cell, pre-mature neuron and mature neurons respectively (Heng et al., 2014), suggesting the neural differentiation potential of hUC-MSCs. RES could facilitate neural-lineage development of hUC-MSCs in a dose-dependent manner, as evidenced by morphological characters and up-regulated expression of $\beta$ III-tubulin and NSE with down-regulated Nestin. Simultaneously, the expression of proneural basic helix loop helix (bHLH) transcription factors, Ngn1, Ngn2 and Mash1 changed. Ngn1 was significantly down-regulated, while Ngn2 and Mash1 were up-regulated in the differentiated hUC-MSCs, and that could be further enhanced by pre-incubation with 2.5, 5 and 10 $\mu \mathrm{M}$ RES. In light with previous studies, Ngn1 is a proneural bHLH transcription factor expressed in newly committed neuronal progenitors and immature neurons and plays an essential role in neurogenesis. It is expressed at a high level in the early stage and decreased in the late stage of neuronal differentiation of MSCs (Cardozo et al., 2012). Ngn2 and Mash1 are activatortype bHLH genes essential for neuronal fate determination (Cardozo et al., 2012; Yu et al., 2013). This study reveals that RES facilitates neuronal differentiation through induction of proneural transcription factor genes in hUC-MSCs, and suggests that RES pretreatment of hUC-MSCs might be beneficial to stem cell based therapy for neural system injury or diseases.

We sought to elucidate the mechanistic basis for these observations and found that RES could regulate the SIRT1 signaling in the hUC-MSCs, which was closely related with cell selfrenewal (Liu et al., 2012), senescence (Chen et al., 2014; Rehan et al., 2014), apoptosis (Godoy et al., 2014) and neural differentiation (Hisahara et al., 2008). Consisted with previous studies (Rathbone et al., 2009), the expression of PCNA, a marker of S-phase to detect the DNA duplication and cell proliferation, was closely related with SIRT1. The up-regulated expression of SIRT1 and PCNA after 0.1, 1 and $2.5 \mu \mathrm{M}$ RES 
treatment was the underlying mechanism for the cell proliferation boost. Moreover, it was revealed that the activity and halflife of $p 53$ could be reduced by SIRT1, and result in increase in cell survival and decrease in cell apoptosis under various DNAdamaging conditions (Hubbard and Sinclair, 2014). Additionally, in line with the report (Chen et al., 2014) that the level of p16 was up-regulated with decreased SIRT1 expression and contribute to cell senescence and cell cycle arrest (da Luz et al., 2012; Vassallo et al., 2014), the impressed expression of SIRT1 in hUC-MSCs by 5 and $10 \mu \mathrm{M}$ RES, along with the enhanced p53 and p16 result in cell senescence, cell cycle arrest and apoptosis. Taken together, the effect of RES on the fate of hUCMSCs is mediated, at least in part, by Sirt1 signaling. However, it is also important to note that, gene expression could be regulated by various factors in a complex way, and the specific roles of SIRT1 may depend on the SIRT1 level, activity, subcellular localization, promoter occupancy and predominant substrates of SIRT1 in the specific microenvironment of different cells (da Luz et al., 2012). Therefore, the controversy in different literatures regarding the effects of RES and SIRT1 could be explained by the response of specific tissues and cells (Chen et al., 2014; Kumazaki et al., 2013; Marambaud et al., 2005; Saharan et al., 2013) and caution should be taken before establishing links between SIRT1 activation and neural protection. Moreover, it is provoking to study the cross-talk between SIRT1 signaling and many other signaling pathways, such as the Wnt/ß-catenin, PI3K/AKT (Tsai et al., 2013), ER-ERK1/2 (Dai et al., 2007), ER/NO/cGMP (Song et al., 2006), etc.

Herein, we suggest future studies on the electrophysiology of the neural-like cells, which would further confirm the effects of RES on the function of the neural differentiated hUC-MSCs. Effects of prolonged RES exposure also need to be explored in order to analyze the duration-dependent effect of RES on the biology of hUC-MSCs. Moreover, researches on animals models are warranted to facilitate the clinical application of RES premodified hUC-MSCs in treating Alzheimer' s disease, Parkinson, stroke, trauma induced brain injury and other neurodegenerative and neural injury disorders.

\section{CONCLUSION}

The present study indicates that RES exerts concentrationdependent effects on the fate of hUC-MSCs in vitro by regulating cell viability, proliferation, senescence, cell cycle, apoptosis and neural-lineage differentiation through activation of SIRT1 signaling. It highlights the promising application of RES in MSCs-based therapy in neuro-regenerative medicine and encourages further exploration on the role of RES in the development of stem cells to meet specific purposes in scientific research and clinical application.

\section{ACKNOWLEDGMENTS}

This study was supported by the National Natural Science Foundation of China (81471306, U1404313), the Innovative Research Team (in Science and Technology) of the University of Henan Province (15IRTSTHN022), the Plan For Scientific Innovation Talent of Henan Province (154200510008). We thank Kristyn Gumpper, Kuncheng Song et al. in Dr. Jianjie Ma's lab and all the team members in Dr. Guan's lab for their discussion and suggestion on the manuscript.

\section{REFERENCES}

Atkins, K.M., Thomas, L.L., Barroso-Gonzalez, J., Thomas, L.,
Auclair, S., Yin, J., Kang, H., Chung, J.H., Dikeakos, J.D., and Thomas, G. (2014). The multifunctional sorting protein PACS-2 regulates SIRT1-mediated deacetylation of p53 to modulate p21dependent cell-cycle arrest. Cell Rep. 8, 1545-1557.

Can, A., and Karahuseyinoglu, S. (2007). Concise review: human umbilical cord stroma with regard to the source of fetus-derived stem cells. Stem Cells 25, 2886-2895.

Cardozo, A.J., Gomez, D.E., and Argibay, P.F. (2012). Neurogenic differentiation of human adipose-derived stem cells: relevance of different signaling molecules, transcription factors, and key marker genes. Gene 511, 427-436.

Chen, H., Liu, X., Chen, H., Cao, J., Zhang, L., Hu, X., and Wang, J. (2014a). Role of SIRT1 and AMPK in mesenchymal stem cells differentiation. Ageing Res. Rev. 13, 55-64.

Chen, H., Liu, X., Zhu, W., Chen, H., Hu, X., Jiang, Z., Xu, Y., Wang L., Zhou, Y., Chen, P., et al. (2014b). SIRT1 ameliorates agerelated senescence of mesenchymal stem cells via modulating telomere shelterin. Front Aging Neurosci. 6, 103.

Chen, B., Zang, W., Wang, J., Huang, Y., He, Y., Yan, L., Liu, J., and Zheng, W. (2015). The chemical biology of sirtuins. Chem. Soc. Rev. 44, 5246-5264.

da Luz, P.L., Tanaka, L., Brum, P.C., Dourado, P.M., Favarato, D., Krieger, J.E., and Laurindo, F.R. (2012). Red wine and equivalent oral pharmacological doses of resveratrol delay vascular aging but do not extend life span in rats. Atherosclerosis 224, 136-142.

Dai, Z., Li, Y., Quarles, L.D., Song, T., Pan, W., Zhou, H., and Xiao, Z. (2007). Resveratrol enhances proliferation and osteoblastic differentiation in human mesenchymal stem cells via ERdependent ERK1/2 activation. Phytomedicine 14, 806-814.

Godoy, J.A., Zolezzi, J.M., Braidy, N., and Inestrosa, N.C. (2014) Role of Sirt1 during the ageing process: relevance to protection of synapses in the brain. Mol. Neurobiol. 50, 744-756.

Guo, R., Li, W., Liu, B., Li, S., Zhang, B., and Xu, Y. (2014). Resveratrol protects vascular smooth muscle cells against high glucose-induced oxidative stress and cell proliferation in vitro. Med. Sci. Monit Basic Res. 20, 82-92.

Heng, B.C., Saxena, P., and Fussenegger, M. (2014). Heterogeneity of baseline neural marker expression by undifferentiated mesenchymal stem cells may be correlated to donor age. J. Biotechnol. 174, 29-33.

Hisahara, S., Chiba, S., Matsumoto, H., Tanno, M., Yagi, H., Shimohama, S., Sato, M., and Horio, Y. (2008). Histone deacetylase SIRT1 modulates neuronal differentiation by its nuclear translocation. Proc. Natl. Acad. Sci. USA 105, 1559915604.

Hubbard, B.P., and Sinclair, D.A. (2014). Small molecule SIRT1 activators for the treatment of aging and age-related diseases. Trends Pharmacol. Sci. 35, 146-154.

Ido, Y., Duranton, A., Lan, F., Weikel, K.A., Breton, L., and Ruderman, N.B. (2015). Resveratrol prevents oxidative stressinduced senescence and proliferative dysfunction by activating the AMPK-FOXO3 cascade in cultured primary human keratinocytes. PLoS One 10, e0115341.

Jackson, S.J., Singletary, K.W., Murphy, L.L., Venema, R.C., and Young, A.J. (2016). Phytonutrients differentially stimulate $\mathrm{NAD}(\mathrm{P}) \mathrm{H}$ :quinone oxidoreductase, inhibit proliferation, and trigger mitotic catastrophe in Hepa1c1c7 cells. J. Med. Food 19, 47-53.

Joe, I.S., Jeong, S.G., and Cho, G.W. (2015). Resveratrol-induced SIRT1 activation promotes neuronal differentiation of human bone marrow mesenchymal stem cells. Neurosci. Lett. 584, 97102.

Karahuseyinoglu, S., Cinar, O., Kilic, E., Kara, F., Akay, G.G., Demiralp, D.O., Tukun, A., Uckan, D., and Can, A. (2007). Biology of stem cells in human umbilical cord stroma: in situ and in vitro surveys. Stem Cells 25, 319-331.

Kumazaki, M., Noguchi, S., Yasui, Y., Iwasaki, J., Shinohara, H. Yamada, N., and Akao, Y. (2013). Anti-cancer effects of naturally occurring compounds through modulation of signal transduction and miRNA expression in human colon cancer cells. J. Nutr. Biochem. 24, 1849-1858.

Lee, J.K., Jin, H.K., Endo, S., Schuchman, E.H., Carter, J.E., and Bae J.S. (2010). Intracerebral transplantation of bone marrow-derived mesenchymal stem cells reduces amyloid-beta deposition and rescues memory deficits in Alzheimer's disease mice by modulation of immune responses. Stem Cells 28, 329-343. 
Liu, B., Ghosh, S., Yang, X., Zheng, H., Liu, X., Wang, Z., Jin, G., Zheng, B., Kennedy, B.K., Suh, Y., et al. (2012). Resveratrol rescues SIRT1-dependent adult stem cell decline and alleviates progeroid features in laminopathy-based progeria. Cell Metab. 16, 738-750.

Luo, J., Nikolaev, A.Y., Imai, S., Chen, D., Su, F., Shiloh, A., Guarente, L., and Gu, W. (2001). Negative control of p53 by Sir2alpha promotes cell survival under stress. Cell 107, 137-148.

Ma, S., Liang, S., Jiao, H., Chi, L., Shi, X., Tian, Y., Yang, B., and Guan, F. (2014). Human umbilical cord mesenchymal stem cells inhibit C6 glioma growth via secretion of dickkopf-1 (DKK1). Mol. Cell Biochem. 385, 277-286.

Marambaud, P., Zhao, H., and Davies, P. (2005). Resveratrol promotes clearance of Alzheimer's disease amyloid-beta peptides. J. Biol. Chem. 280, 37377-37382.

Mikula-Pietrasik, J., Kuczmarska, A., Rubis, B., Filas, V., Murias, M. Zielinski, P., Piwocka, K., and Ksiazek, K. (2012). Resveratrol delays replicative senescence of human mesothelial cells via mobilization of antioxidative and DNA repair mechanisms. Free Radic. Biol. Med. 52, 2234-2245.

Ozcan, P., Ficicioglu, C., Yildirim, O.K., Ozkan, F., Akkaya, H., and Aslan, I. (2015). Protective effect of resveratrol against oxidative damage to ovarian reserve in female Sprague-Dawley rats. Reprod. Biomed. Online 31, 404-410.

Park, H.R., Kong, K.H., Yu, B.P., Mattson, M.P., and Lee, J. (2012). Resveratrol inhibits the proliferation of neural progenitor cells and hippocampal neurogenesis. J. Biol. Chem. 287, 42588-42600.

Pinarli, F.A., Turan, N.N., Pinarli, F.G., Okur, A., Sonmez, D., Ulus, T., Oguz, A., Karadeniz, C., and Delibasi, T. (2013). Resveratrol and adipose-derived mesenchymal stem cells are effective in the prevention and treatment of doxorubicin cardiotoxicity in rats. Pediatr. Hematol. Oncol. 30, 226-238.

Rathbone, C.R., Booth, F.W., and Lees, S.J. (2009). Sirt1 increases skeletal muscle precursor cell proliferation. Eur. J. Cell Biol. 88, 35-44.

Rehan, L., Laszki-Szczachor, K., Sobieszczanska, M., and PolakJonkisz, D. (2014). SIRT1 and NAD as regulators of ageing. Life Sci. 105, 1-6.

Rimmele, P., Lofek-Czubek, S., and Ghaffari, S. (2014). Resveratrol increases the bone marrow hematopoietic stem and progenitor cell capacity. Am. J. Hematol. 89, E235-238.

Saharan, S., Jhaveri, D.J., and Bartlett, P.F. (2013). SIRT1 regulates the neurogenic potential of neural precursors in the adult subventricular zone and hippocampus. J. Neurosci. Res. 91, 642-659.

Simic, P., Zainabadi, K., Bell, E., Sykes, D.B., Saez, B., Lotinun, S.,
Baron, R., Scadden, D., Schipani, E., and Guarente, L. (2013). SIRT1 regulates differentiation of mesenchymal stem cells by deacetylating beta-catenin. EMBO Mol. Med. 5, 430-440.

Song, L.H., Pan, W., Yu, Y.H., Quarles, L.D., Zhou, H.H., and Xiao, Z.S. (2006). Resveratrol prevents CsA inhibition of proliferation and osteoblastic differentiation of mouse bone marrow-derived mesenchymal stem cells through an ER/NO/cGMP pathway. Toxicol. In Vitro 20, 915-922.

Tsai, J.H., Hsu, L.S., Lin, C.L., Hong, H.M., Pan, M.H., Way, T.D., and Chen, W.J. (2013). 3,5,4'-Trimethoxystilbene, a natura methoxylated analog of resveratrol, inhibits breast cancer cell invasiveness by downregulation of PI3K/Akt and Wnt/betacatenin signaling cascades and reversal of epithelialmesenchymal transition. Toxicol. Appl. Pharmacol. 272, 746-756.

Vassallo, P.F., Simoncini, S., Ligi, I., Chateau, A.L., Bachelier, R. Robert, S., Morere, J., Fernandez, S., Guillet, B., Marcelli, M., et al. (2014). Accelerated senescence of cord blood endothelial progenitor cells in premature neonates is driven by SIRT1 decreased expression. Blood 123, 2116-2126.

Yu, Q., Liu, L., Duan, Y., Wang, Y., Xuan, X., Zhou, L., and Liu, W. (2013). Wnt/beta-catenin signaling regulates neuronal differentiation of mesenchymal stem cells. Biochem. Biophys. Res. Commun. 439, 297-302.

Yuan, H.F., Zhai, C., Yan, X.L., Zhao, D.D., Wang, J.X., Zeng, Q. Chen, L., Nan, X., He, L.J., Li, S.T., et al. (2012). SIRT1 is required for long-term growth of human mesenchymal stem cells. J. Mol. Med. (Berl). 90, 389-400.

Zhang, D.Y., Wang, H.J., and Tan, Y.Z. (2011). Wnt/beta-catenin signaling induces the aging of mesenchymal stem cells through the DNA damage response and the p53/p21 pathway. PLoS One 6, e21397.

Zhang, T., Tian, F., Wang, J., Zhou, S., Dong, X., Guo, K., Jing, J., Zhou, Y., and Chen, Y. (2015). Donepezil attenuates high glucose-accelerated senescence in human umbilical vein endothelial cells through SIRT1 activation. Cell Stress Chaperones 20, 787-792.

Zhu, Y., He, W., Gao, X., Li, B., Mei, C., Xu, R., and Chen, H. (2015). Resveratrol overcomes gefitinib resistance by increasing the intracellular gefitinib concentration and triggering apoptosis, autophagy and senescence in PC9/G NSCLC cells. Sci Rep. 5, 17730

Zhu, X., Zhang, Y., Li, Q., Yang, L., Zhang, N., Ma, S., Zhang, K., Song, J. and Guan, F. (2016). $\beta$-carotene induces apoptosis in human esophageal squamous cell carcinoma cell lines via the Cav-1/AKT/NF-kappaB signaling pathway. J. Biochem. Mol. Toxicol. 30, 148-157. 